\title{
Cost Analysis of Handover Manager Based Handover Method in LEO Satellite Networks
}

\author{
Kousik Maity ${ }^{1}$, Soumya Das ${ }^{1}$,Arumoy Saha ${ }^{2}$, Bhaskar pal ${ }^{1}$ and Arkodyuti \\ Sarkar \\ 1Department of Electronics and Communication Engg, Bengal Institute of Technology, WBUT, India, Kolkata- \\ 700150. \\ 2 Department of Information Technology, Bengal Institute of Technology, WBUT, India,Kolkata - 700150. \\ 3Department of Computer Science and Engg, Bengal Institute of Technology, WBUT, India,Kolkata-700150.
}

\begin{abstract}
LEO satellite has an important role in global communication system. They have advantages like low power requirement and lower end-to-end delay, efficient frequency spectrum utilization between satellites and spotbeams over MEO and GEO satellites. So in future they can be used as a replacement of modern terrestrial wireless networks. There are a lot of handover techniques for LEO satellites like seamless handover (SeaHOLEO), PatHO-LEO. In our previous work, we have suggested a new handover technique for SeaHO-LEO by introducing a Handover Manager (HM) during the handover process and by simulation we have also shown that it a better approach by comparing it with other existing handover techniques as it reduces the handover latency, propagation delay, call blocking probability more than any other technique. In this paper we have evaluated the exact cost of our previous work i.e. Handover Manager based handover Method (HMBHO). Simulation results show that the cost of Handover Manager based handover management method is better than other handover methods.
\end{abstract}

Keywords: Handover latency, LEO, Mobile Node (MN),Handover Manager (HM).

\section{Introduction:}

Satellite communication networks are utilized to co exist with terrestrial networks to provide global coverage to a heterogeneously distributed over population,. A LEO satellite takes about 100 minutes to orbit the earth, which means that a single satellite is "in view" of ground equipment for only a few minutes [1]. As a consequence, if a transmission takes more than the short time period than any one satellite.

\section{Handover:}

In cellular telecommunications, the term handover or handoff refers to the process of transferring an ongoing call or data session from one channel connected to the core network to another. In satellite communications it is the process of transferring satellite control responsibility from one earth station to another without loss or interruption of service.

\section{Types of handover}

A Hard Handover is one in which the channel in the source cell is released and only then the channel in the target cell is engaged. Thus the connection to the source is broken before or 'as' the connection to the target is made - for this reason such handovers are also known as break-before-make. Hard handovers are intended to be instantaneous in order to minimize the disruption to the call. When the mobile is between base stations, then the mobile can switch with any of the base stations, so the base stations bounce the link with the mobile back and forth.

A Soft Handover is one in which the channel in the source cell is retained and used for a while in parallel with the channel in the target cell. In this case the connection to the target is established before the connection to the source is broken, hence this handover is called make-before-break. The interval, during which the two connections are used in parallel, may be brief or substantial. Soft handovers may involve using connections to more than two cells: connections to three, four or more cells can be maintained by one phone at the same time. The latter is more advantageous, and when such combining is performed both in the downlink (forward link) and the uplink (reverse link) the handover is termed as softer. Softer handovers are possible when the cells involved in the handovers have a single cell site.

A LEO satellite system must hand over between satellites to complete the transmission. In general, this can be accomplished by constantly relaying signals between the satellite and various ground stations, or by communicating between the satellites themselves using "inter-satellite links"(ISLs) [1], [2]. LEO satellites are also designed to have more than one satellite in view from any spot on the earth at any given time, minimizing the possibility that the network will loose the transmission. Due to the fast-flying satellites, LEO systems must 
incorporate complicated tracking and switching equipment to maintain consistent service coverage. In this paper, we focus on the handover management of satellite networks, which is a crucial design problem for supporting mobile communication services in the co-existing terrestrial and LEO satellite networks.

One of the proposed models for handover management in satellite networks is mobile IP (MIP) [3]. When a mobile host moves from one point of attachment to another it enables a TCP connection to remain alive and to continue receiving packets. Although MIP is a widely used approach applied to satellite networks, it has some important drawbacks including high handover latency and high packet loss [4][5].

In our paper we have proposed an idea of reducing binding updates, handover latency and packet loss during handover. Our paper is structured as follows:

In the first section we have given a brief introduction related to LEO satellite and handover mechanisms. In section 2 we have discussed the related work regarding MIP network and our previous work named Handover Manager Based Handover method (HMBHO). In next section we have proposed the cost analysis of the HMBHO. In section $4 \& 5$ we have shown the simulation results and the conclusion and the future work regarding this paper.

\section{Related Work:}

The most widely used protocol for handover in satellite is MIP [7]. It is proposed by The Internet engineering task force (IETF) to handle mobility of internet hosts for mobile data communications. MIP is based over the concept of Home Agent (HA) and Foreign Agent (FA) for delivering of packets from one MN to CN. It is basically completed by four steps.

i) When handover begins MN registers itself in FA and waits for allocation of channels in FA and updates its location in HA directory.

ii) The packets are sent to HA and HA encapsulate it.

iii) Encapsulated packets are sent to The FA.

iv) FA decapsulate those packets and sent it to MN.

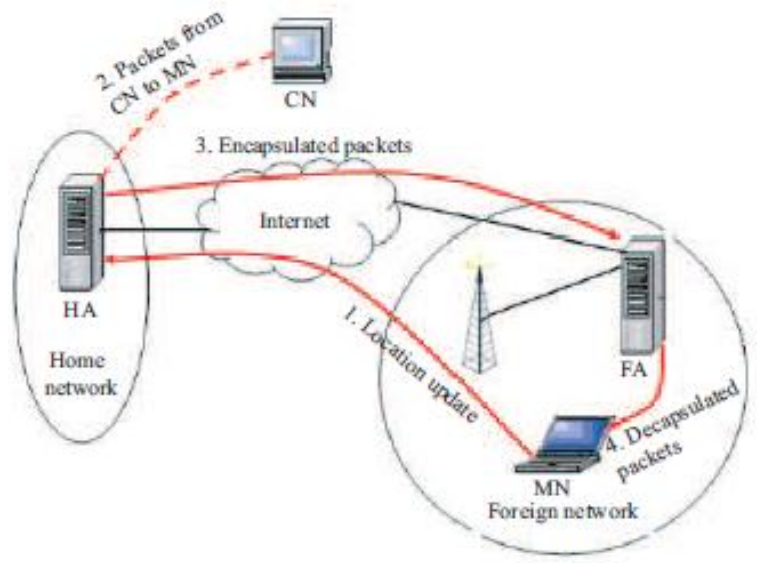

Figure 1: Handover Flow of Mobile IP

The main drawback of this protocol is
a. High handover latency
b. High packet lost rate
c. Insufficient routing path
d. Conflicts with network security solution

Another method is Seamless handover management scheme (SEAHO-LEO) [8],[9] proposed by Aysegul et al in 2006.

It reduces packet loss and handover latency. It is describes as follows
A. Calculate a new IP
B. Send handover preparation request to current satellite
C. Start to use new IP to send data packets
D. CN starts to use new satellite

SEAHO-LEO provides efficient utilization of network bandwidth because of the absence of tunneling and also does not need any change in existing internet infrastructure.

The main disadvantage of this process is high messaging traffic. 
Another method is Seamless handover management scheme (SeaHO-LEO) [14], [15] proposed by Aysegul et al in 2006.

It reduces packet loss and handover latency. It is describes as follows

A. Calculate a new IP

B. Send handover preparation request to current satellite

C. Start to use new IP to send data packets

D. CN starts to use new satellite

SeaHO-LEO provides efficient utilization of network bandwidth because of the absence of tunnelling and also does not need any change in existing internet infrastructure.

The main disadvantage of this process is high messaging traffic.

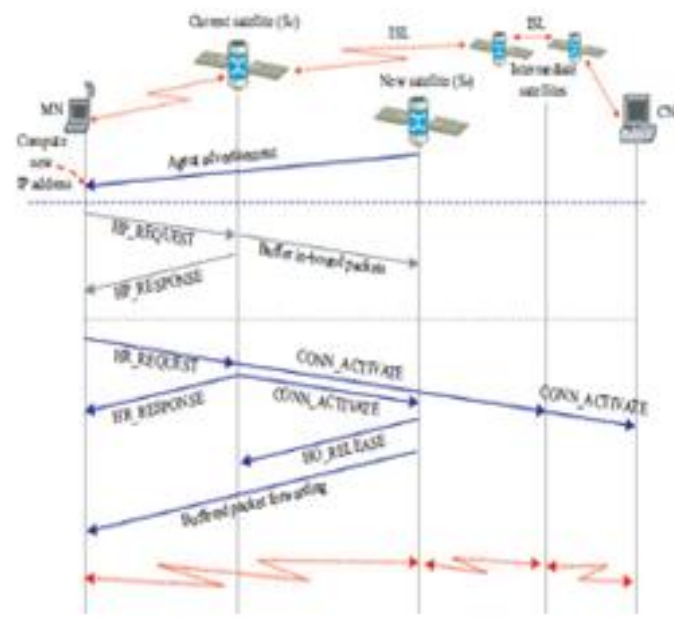

Figure 4: Signalling Flow of SeaHO-LEO

In our previous work [16] we have introduced a new Handover Manager (HM) which will reduce the binding updates and handover latency as it shows that there is no need to send and receive handover preparation request prior to handover. so, the process of the handover of the SeaHO-LEO is just as follows:

1) First initiates the process by searching for the first IP or the new IP when the MN moves into the overlapping area of two adjacent satellite footprints, determining the signal strength and the QOS parameter choose the ideal one and register it.

Find out the next IP where the packets need to be forwarded by measuring the signal strength of the satellites while the satellite is moving in a network. The IP address of two adjacent satellite is stored in handover manager for the fraction of second until the next IP arises and by comparing the signal strength it will keep the IP of maximum signal strength.

2) Now when the handover is needed there is no need to send handover preparation or the handover response request as the IP is already stored in the handover manager(HM).

3) Handover will be initiated.

4) The data packets will be forwarded to the new ip stored in HM .

5) During the forwarding of packets HM will keep searching for the IP and when the first data forwarding will be completed it will start sending to the next IP stored.

6) Thus the process continues.

\section{Flowchart:}

The flowchart of the proposed handover has been shown in the fig 3 . 


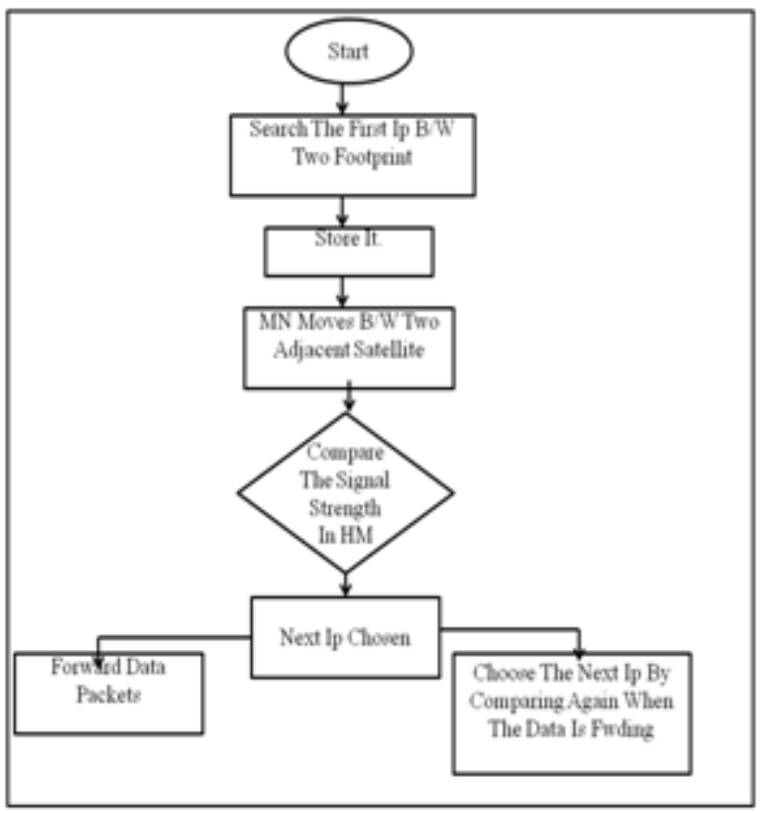

Fig 3 Flow chart of this proposed method:

It will reduce the binding updates as well as handoff latency as there is no need to register for the handoff preparation request and handoff response request. Introduction of HM will both the thing itself. Also there is no extra searching time for the new IP. It will find it while the MN will move to different ips by comparing the signal strength.

\section{Proposed Work:}

In our previous work we have discussed the HMBHO method and shown how it can reduce handover latency and call blocking probability.

Here we will analysis the cost of this HMBHO method and compare it with other standard methods like MIP.

\section{Mobility Management Cost Definition}

In [12] the mobility management cost is evaluated as the product of generated control message size, $\mathrm{M}$ and the number of hopes, $\mathrm{H}$, required to deliver the message. If we apply such definition into the paging cost, it will be proportional with the number of receivers. Taking into account the broadcasting capabilities of satellites, however, the cost is also simply a product of the message size and the number of travelled hops.

\section{Costs of different Mobility management events:}

$$
\text { Cost=M.H }
$$

The following defines the cost required for each mobility management event; binding update, local forwarding and paging

For each case, the Control messages generated are assumed to be equally sized (M) in all the four events. The number of control messages that are generated upon a handover occurrence between mobile nodes and the corresponding ARs, is assumed to be same for MIP and our proposed method. Thus we can neglect the number of control message in the cost evaluation.

1. Binding Update Cost: Let $H_{M N, L D}$ denote the number of hops between a mobile node and the Location Directory. The cost for binding update procedure can be expressed as:

$$
\text { M. } \mathrm{H}_{\mathrm{MN}, \mathrm{LD}}
$$

2. Local Forwarding Cost: Denoting the number of hops between two adjacent satellites as $H_{A R, A R}$ the local forwarding cost is shown as follows:

$$
M \cdot H_{A R, A R}
$$

\section{Management Cost of MIP and our proposed method}

The costs of Mobile IP and our proposed method are as follows

A. Mobile IP: The cost of MIP is the product of binding update cost and rate of handover occurrence. The local forwarding, paging and GPS are not used here. So the MIP management cost, $\mathrm{C}_{\mathrm{MIP}}(\mathrm{t})$ can be expressed as

Where the rate of handover occurrence, $R_{H O}(t)$, is:

$$
\mathrm{C}_{\mathrm{MIP}}(\mathrm{t})=\mathrm{M} \cdot \mathrm{H}_{\mathrm{MN}, \mathrm{LD}} \cdot \mathrm{R}_{\mathrm{HO}}(\mathrm{t})
$$




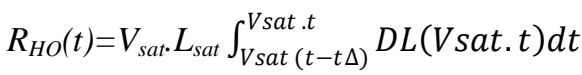

Where, $\mathrm{V}_{\text {sat }}$ and $\mathrm{L}_{\mathrm{sat}}$ denote the ground speed of satellite and the coverage boundary length, respectively. $D_{L}\left(V_{\text {sat }} . t\right)$ denote the linear density of nodes on the coverage boundary at time $t$.

B. PatHO-LEO: In the PatHO-LEO model, the local forwarding and paging scheme create some additional cost. The total cost of PatHO-LEO model $\mathrm{C}_{\text {PatHO-LEO }}(\mathrm{t})$ is

$$
\mathrm{C}_{\mathrm{PatHO}-\mathrm{LEO}}(\mathrm{t})=\mathrm{M} \cdot \mathrm{H}_{\mathrm{MN}, \mathrm{LD}}+\mathrm{M} \cdot \mathrm{H}_{\mathrm{AR}, \mathrm{AR}} \mathrm{R}_{\mathrm{HO}}(\mathrm{t}) \cdot \alpha+\left\{\mathrm{M} \cdot \mathrm{H}_{\mathrm{AR}, \mathrm{AR}}(\mathrm{S}-1)+\mathrm{M} \cdot \mathrm{S}\right\}^{*} \mathrm{n}(\mathrm{t})(1-\alpha) \cdot \lambda
$$

Where,. $\mathrm{H}_{\mathrm{AR}, \mathrm{AR}}$ and $\mathrm{S}$ denote the number of hops between two adjacent satellites and the number of single-beam satellites that cover a single paging area, respectively. $\mathrm{n}(\mathrm{t})$ and $\alpha$ denote the total number of MNs per a coverage area at time $\mathrm{t}$ and the ratio of active MNs to the total number of MNs, respectively. The rate of new connections to a $\mathrm{MN}$ is denoted as $\lambda$.

C. HMBHO Method: In our proposed Handover Manager Based Handover Method we have induced a handover manager to reduce the cost. Now there will no extra cost of sending handover preparation request or the handover response request. So the number of messages exchanged between will be only two as to send the handover request and to receive the resulted satellite IP. So the cost of the message exchanged between MN and $\mathrm{HM} \mathrm{C}_{\mathrm{MN}, \mathrm{HM}}$ is

$$
\mathrm{C}_{\mathrm{MN}, \mathrm{HM}}=2 * \mathrm{M}^{*} \mathrm{H}_{\mathrm{MN}, \mathrm{HM}}
$$

Next the total number of message exchanged between satellite and the Handover Manager will be based upon the number of satellite seen by the Handover manager that time. So the So the cost of the message exchanged between Satellite and $\mathrm{HM} \mathrm{C}_{\mathrm{Sat}, \mathrm{HM}}$ is

$$
\mathrm{C}_{\mathrm{Sat}, \mathrm{HM}}=\mathrm{n} * \mathrm{M} * \mathrm{H}_{\mathrm{Sat}, \mathrm{HM}}
$$

Where $\mathrm{n}$ is the number of satellite seen by that time by the Handover Manager.

So the total cost of messaging will be $\mathrm{C}_{\mathrm{MSG}}$ is

So the total cost of our proposed work is

$$
\mathrm{C}_{\mathrm{MSG}}=\mathrm{C}_{\mathrm{MN}, \mathrm{HM}}+\mathrm{C}_{\text {Sat }, \mathrm{HM}}
$$

$$
\begin{aligned}
\mathrm{C}_{\mathrm{Tot}} & =\left(\mathrm{C}_{\mathrm{MSG}}+\mathrm{M} \cdot \mathrm{H}_{\mathrm{AR}, \mathrm{AR}}\right) * \mathrm{R}_{\mathrm{HO}}(\mathrm{t}) \\
& =\left(\mathrm{C}_{\mathrm{MN}, \mathrm{HM}}+\mathrm{C}_{\mathrm{Sat}, \mathrm{HM}}+\mathrm{M} . \mathrm{H}_{\mathrm{AR}, \mathrm{AR}}\right) * \mathrm{R}_{\mathrm{HO}}(\mathrm{t}) \\
& =\left(2 * \mathrm{M} * \mathrm{H}_{\mathrm{MN}, \mathrm{HM}}+\mathrm{n} * \mathrm{M} * \mathrm{H}_{\mathrm{Sat}, \mathrm{HM}} \quad \mathrm{M} . \mathrm{H}_{\mathrm{AR}, \mathrm{AR}}\right) * \mathrm{R}_{\mathrm{HO}}(\mathrm{t})
\end{aligned}
$$

Equation 8 represents the total cost of HMBHO method.

\section{Simulation Results:}

In order to evaluate the performance HANDOVER MANAGER with SeaHO-LEO, we compared it to MIP \& SeaHO-LEO scheme. Each algorithm is evaluated by analyzing the Handoff delay, Forced call termination probability \& Handover latency. The simulation results have been shown using the MATLAB 7.8 in a designed virtual environment.

In figure 4 we compare the Handover throughput for MIP, SeaHO-LEO \& Handover Manager i.e. the proposed SEAHO-LEO handover throughput during a handover process. In mobile IP, due to the tunneling between HA and FA, throughput of the channel between $\mathrm{MN} 1 / \mathrm{CN}$ and $\mathrm{MN} 2 / \mathrm{MN}$ converges to zero during handover. When the handover model is completed, the throughput reaches a reasonable value. The throughput of SeaHO-LEO is better than MIP during handover as it does not reach to zero. In BMBHO the throughput is higher than SeaHO-LEO because the handover takes very less time and the packets during handover is sent by the old link.

In MIP the MN has to search for a new satellite \& then analyze them. For SeaHO-LEO the handoff delay is closer to MIP but in our work as everything is kept within BM \& BM just have to run a simple algorithm so the handoff delay is very much less.

In figure 5 we compare the Forced call termination probability of MIP \& SeaHO-LEO with the proposed SeaHO-LEO. Among this three handover management models, HANDOVER MANAGER i.e. our new proposed seaHO-LEO has the lowest Forced call termination probability. In MIP the MN has to wait for the channel allocation \& if it did not get a free channel within the handoff time the call is being terminated. In SeaHO-LEO the MN has to wait for the agent advertisement from a new satellite. If it did not get it within handoff time the call is being 


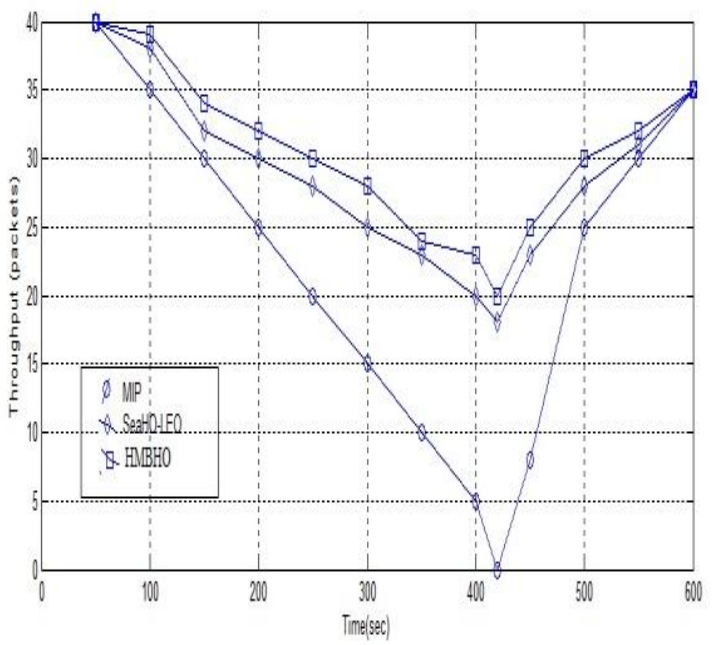

Figure 4: Simulation results of MN's handover throughput

terminated. But in HM there is no need to wait as it initatializes the next call earlier during the first packet forwarding by comparing the adjacent satellite's signal strength .Hence the call blocking probability is very less.

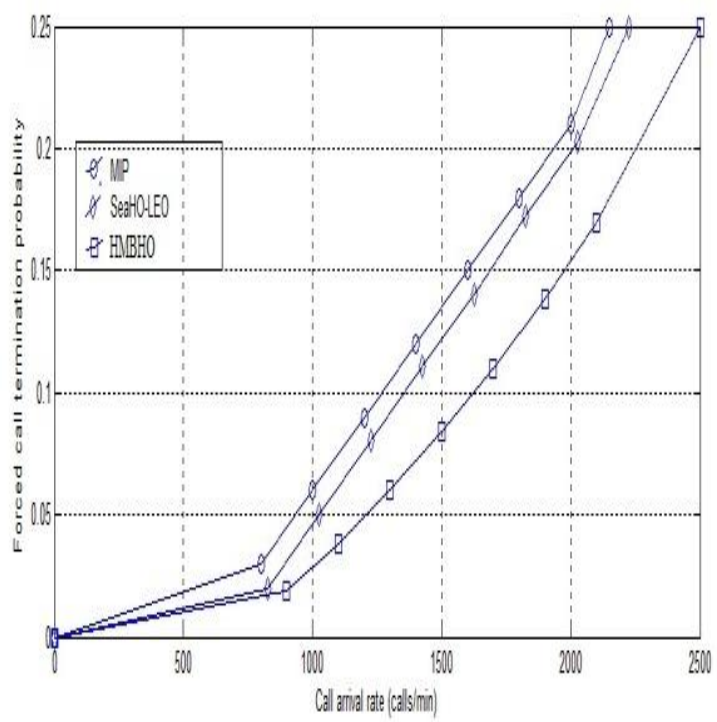

Figure 5: Forced call termination probability of a handover call

In figure 6 we have shown the average handoff latency. Handoff latency affects the service quality of real time applications of mobile users. It is dependent on the time taken to establish a new path segment between MN and new satellite. Basically it is the time interval between the last data segment received through the old path and the first data segment received through the new path from CN/MN2 to MN/MN1.

In MIP, the handover latency is immense because MN has to send location update message to it's HA to associate its home address and CoA. This binding update process is time consuming operation. MIP is incapable of receiving packet in flight during registration process. In the case of MIP, the MN always uses its home address to send and receive packets, and it cannot contact the old FA (satellite) we can see there is a transmission stall of about $240 \mathrm{~ms}$, which represents the handover latency when using MIP. This handover latency is independent from the time spent in overlapping area of new and old satellite. In SeaHO-LEO, as soon as the old Satellite receives HR_REQUEST message it establishes a virtual communication path between the new satellite and MN by the help of ISL between it and new satellite. This process needs approximately $20 \mathrm{~ms}$. In our proposed method there is no need to send or receive HR_REQUEST and no need to wait as the HM itself initiates every handover. That is why the handover latency is very less in our proposed method. 


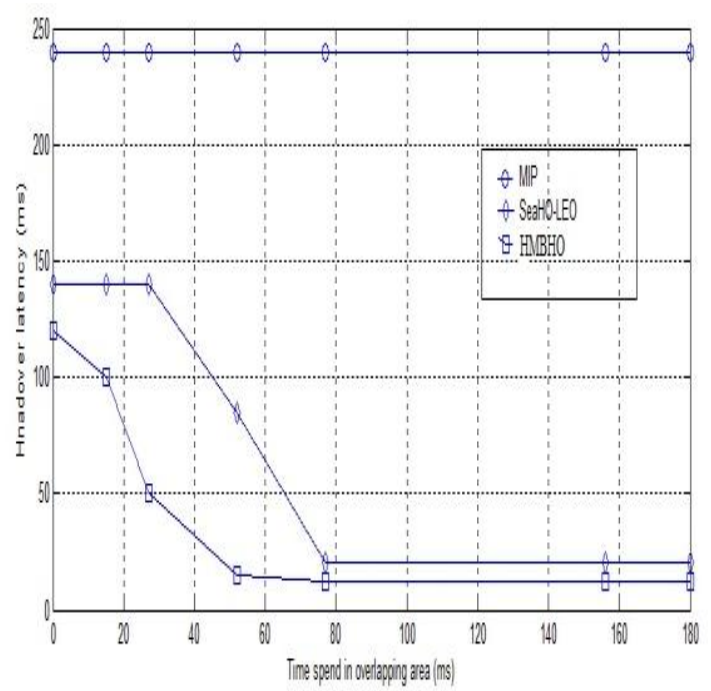

Figure 6: Handover latency

\section{Conclusions:}

In this paper we have evaluated our previously proposed a HANDOVER MANAGER concept to modify the handover technique of SeaHO-LEO satellite handover management where we have shown that our HMBHO method is more effective than the existing one by also showing theoretically as well as by simulation that it reduces handover latency, data loss, scanning time, cost and forced call termination probability. Our HMBHO handover method is also better as compare to the time delay and the binding updates as it itself compares the signal strength and chooses the next IP where the packets are to be forwarded it has reduced the extra binding updates and as the search is done when the previous data is being forwarded it also reduces the searching time after the first data sent. So comparing all the aspects our work is practically applicable to any areas. The cost analysis and simulation result shows that the cost is lower than the related algorithms.

\section{Future Work:}

In future we will try to reduce the cost of $\mathrm{HMBHO}$ method.

\section{References}

[1] S. L. Kota, P. A. Leppanen, and K. Pahlavan, Broadband Satellite Communications For Internet Access, Kluwer Academic Publishers, 2004.

[2] A. Jamalipour, "Satellites in IP networks," in Wiley Encyclopedia ofTelecommunications, vol. 4, Wiley, 2002, pp. 2111-2122.

[3] Satellite Mobility Pattern Scheme for Centrical and Seamless Handover Management in LEO SatelliteNetworks Ays, eg ul T"uys"uz and Fatih Alag"oz

[4] H. Uzunalioglu, I. F. Akyildiz, Y. Yesha, and W. Yen, "Footprint handover

[5] H. Uzunalioglu, I. F. Akyildiz, Y. Yesha, and W. Yen, "Footprint handover Rerouting protocol for low earth orbit satellite networks," Wireless Networks, vol. 5, no. 5, pp. 327-337, 1999

[6] Systems By Joydeep Banerjee D Sarddar, S.K. Saha, M.K. Naskar, T.Jana, U. Biswas

[7] H. N. Nguyen, S. Lepaja, J. Schuringa, and H. R. Van As, "Handover management in low earth orbit satellite IP networks," IEEE Global Telecommunications Conference, San Antonio, TX, USA, pp. 2730-2734, 25-29 November 2001

[8] J. T. Malinen and C.Williams, "Micromobility taxonomy," Internet Draft,IETF, Nov. 2001

[9] T"uys"uz and F. Alag"oz, "Satellite mobility pattern based handover management algorithm in LEO satellites," in Proc. IEEE ICC 2006, Istanbul,Turkey, June 2006.

[10] Ays, eg"ul T"uys"uz and Fatih Alag"oz, "Satellite Mobility Pattern Scheme for centrical and Seamless Handover Management in LEO Satellite Networks", JOURNAL OF COMMUNICATIONS AND NETWORKS, VOL. 8, NO. 4, DECEMBER 2006.

[11] M. Atiquzzaman, S. Fu, and W. Ivancic, "TraSH-SN: A transport layer seamless handoff scheme for space networks," in Proc. ESTC 2004, Palo Alto, CA, June 2004.

[12] Debabrata Sarddar, Soumya Das,Dipsikha Ganguly,Sougata Chakraborty,M.k.Naskar, A New Method for Fast and Low Cost Handover in Leo Satellites(International Journal of Computer Applications (0975 - 8887) Volume 37- No.7, January 2012) http://www.ijcaonline.org/archives/volume37/number7/4622-6631

[13] Debabrata Sarddar, Soumya Das,Dipsikha Ganguly,Sougata Chakraborty, M.k.Naskar,A New Method for Controlling Mobility Management Cost of PatHO- LEO Satellite and Mobile IP Network (International Journal of Computer Applications (09758887)Volume37-No.7,January2012 http://www.ijcaonline.org/archives/volume37/number7/4621-6630.

[14] Dipsikha Ganguly, Debabrata Sarddar, Soumya Das, Suman Kumar Sikdar, Sougata Chakraborty and Kunal Hui. Article: Algorithm Based Approach for the Connection Establishment in the Fast Handover in Leo Satellites in BMBHO. International Journal of Computer Applications 44(12):36-42, April 2012. Published by Foundation of Computer Science, New York, USA

[15] Debabrata Sarddar, Dipsikha Ganguly, Soumya Das, Suman Kumar Sikdar, Sougata Chakraborty, Kunal Hui, Shabnam Bandyopadhyay, Kalyan Kumar Das and Sujoy Palit. Article: Cost Analysis of Location Manager based Handover Method for LEO Satellite Networks. International Journal of Computer Applications 45(19):1-6, May 2012. Published by Foundation of Computer Science, New York, USA 
[16] Debabrata Sarddar, Dipsikha Ganguly, Soumya Das, Suman Kumar Sikdar, Sougata Chakraborty, Kunal Hui, Shabnam Bandyopadhyay, Kallyan Kumar Das and Mrinal Kanti Naskar. Article: Introduction of Handover Manager for Better Handover Latency and Handover Throughput during Leo Satellite Handover. International Journal of Computer Applications 42(15):47-52, March 2012. Published by Foundation of Computer Science, New York, USA.

\section{AUTHORS PROFILE}

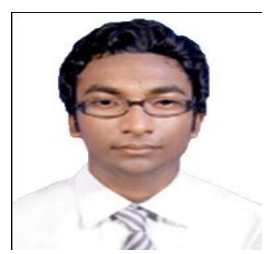

Kousik Maity, currently pursuing B.Tech in Electronics \& Communication Engineering at Bengal Institute of Technology under West Bengal University of Technology. His research interest includes robotics \& wireless Communication.

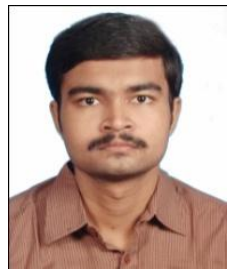

Soumya Das, son of Mr. Subrata Das and Mrs. Swapna Das, currently pursuing his B.Tech in Electronics \& Communication Engg. At Bengal Institute of Technology under West Bengal University of Technology. His research interest includes mobile communication \& satellite communication.

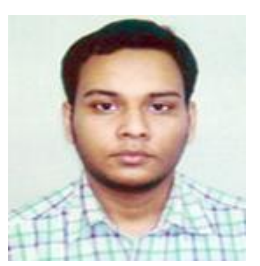

Arumoy Saha, currently pursuing B.Tech in Information Technology at Bengal Institute of Technology under West Bengal University of Technology. His research interest include satellite Communication.

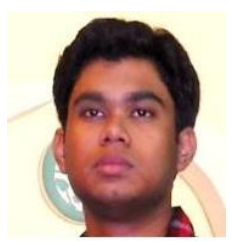

Bhaskar Pal, currently pursuing B.Tech in Electronics \& Communication Engineering at Bengal Institute of Technology under WestBengal University of Technology. His research interest include wireless Communication.

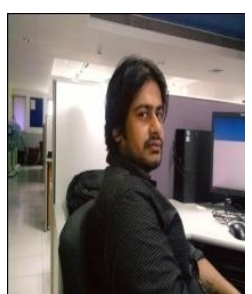

Arkodyuti Sarkar, is presently an employee of TCS. He has completed his B.Tech in C.S.E at Bengal Institute of Technology under West Bengal University of Technology. His research interest includes robotics \& artificial intelligence. 